\title{
Tele-health in neurology: an indispensable tool in the management of the SARS-CoV-2 epidemic
}

\author{
Ruggero Capra $^{1}$ (D) Flavia Mattioli ${ }^{2}$
}

Received: 27 April 2020 / Revised: 30 April 2020 / Accepted: 5 May 2020 / Published online: 12 May 2020

c) Springer-Verlag GmbH Germany, part of Springer Nature 2020

\section{Brief communication}

Since February 2020, SARS-COV 2 pandemic in Lombardy (Italy) progressively limited the access of patients to hospitals because of the risk of contagion. This has generated a need of home health care: tele-health [1], with the aim of monitoring patients' conditions, offering consultations, and carrying out cognitive assessments and rehabilitation.

Here we report examples of tele-health in non-acute neurological patients, which the authors have implemented in Brescia in these last months.

Despite the pressure of COVID-19, multiple sclerosis (MS) management could be continued, either to monitor therapies and the outbreak in the MS population or to treat relapses. Noteworthy, $6.1 \%$ of 1072 MS patients treated with disease-modifying drugs in our MS Center had a SARSCOV-2 infection, with an exponential increase since February 26 till March 26 (from $0.37 \%$ to $5.78 \%$ of the patients' cohort). Therefore, promptly activating remote support was necessary to communicate with patients and support the individuals in critical conditions; this approach resulted to be very appreciated, as 1071 out of 1246 (86\%) who answered to a survey felt they had a comprehensive support. In particular, emails were sent to provide instructions about protection from infection and isolation to prevent familiar contagion; specific emails were sent to patients prescribed with ocrelizumab, alemtuzumab, and cladribine to reinforce their motivation for conscious self-protection. In addition, blood tests and MRI were checked via mail, although direct telephone contact was the patients' preferred mean of relating to health personnel, especially for the management of

Flavia Mattioli

flaviacaterina.mattioli@gmail.com

1 MS Center, Neurology Unit Montichiari Hospital, Via don Ciotti 124 Montichiari, Brescia, Italy

2 Neuropsychology Unit ASST Spedali Civili, Via Nikolajewka, 1325123 Brescia, Italy febrile cases and respiratory symptoms. Maintaining telephone contact with home patients twice a day to record respiratory parameters and the thermal curve was of crucial importance to avoid access to the emergency room and to address only critical cases to hospital care.

Patients with dementia or Parkinson disease were also treated from remote, even if for them the age-related lack of familiarity with electronical devices may impair cognitive testing. Nonetheless, mailing and telephone calls resulted to be essential to monitor and treat behavioral disturbances or delirium in dementia patients and motor deficit in Parkinson disease. Neuropsychological formal evaluations were performed from remote by sharing tests' tools not requiring specific material handling. MS patients, post-stroke, and posttraumatic brain-injured patients with cognitive impairment were tested. Speech and cognitive trainings were provided by prescribing exercises, accessible from the cloud by either the psychologist— to interact with patients' activity—or the patients, often with the help of a caregiver.

Interestingly a higher frequency of aphasia treatments and cognitive rehabilitations per week was accepted by patients (4/week), compared to the traditional setting (usually 2 or $3 /$ week), maybe due to the unnecessary transportation of patients and the greater ease in organization.

Remarkably, a reimbursement mechanism was organized by our Hospital with the regional health system.

The above-mentioned are only examples of tele-health in the neurological field, which we found to be crucial during the COVID-19 emergency. This outbreak changed the usual clinical practice by favoring patients' acceptance of tele-health to maintain contact with neurologists and by allowing patients' clinical sorting before their arrival at the hospital, also in consideration of the reduced availability of health personnel. The opportunity of monitoring and treating patients without moving them often resulted in a higher frequency of contacts with the patients compared to the usual medicine. 
Therefore, well-designed schemes of monitoring, diagnosing, and treatments should be implemented, beyond the COVID-19 emergency, to become part of the new normality rather than the exception, particularly for chronic and vulnerable people and to make the health sector more resilient [2].

\section{Compliance with ethical standards}

Conflicts of interest Authors declare no conflict of interest.

\section{Reference}

1. https://www.who.int/sustainable-development/health-sector/strat egies/telehealth/en/

2. Bloem BR, Dorsey ER, Okun MS (2020) The Coronavirus disease 2019 crisis as catalyst for telemedicine for chronic neurological disorders. JAMA neurology. https://doi.org/10.1001/jamaneurol .2020 .1452 